May 1992

UMDGR-92-208

\title{
THE SPIN HOLONOMY GROUP IN GENERAL RELATIVITY
}

\author{
Ted Jacobson円 and Joseph D. Romano \\ Department of Physics \\ University of Maryland, College Park, MD 20742
}

\begin{abstract}
It has recently been shown by Goldberg et al that the holonomy group of the chiral spin-connection is preserved under time evolution in vacuum general relativity. Here, the underlying reason for the timeindependence of the holonomy group is traced to the self-duality of the curvature 2-form for an Einstein space. This observation reveals that the holonomy group is time-independent not only in vacuum, but also in the presence of a cosmological constant. It also shows that once matter is coupled to gravity, the "conservation of holonomy" is lost.

When the fundamental group of space is non-trivial, the holonomy group need not be connected. For each homotopy class of loops, the holonomies comprise a coset of the full holonomy group modulo its connected component. These cosets are also time-independent. All possible holonomy groups that can arise are classified, and examples are given of connections with these holonomy groups. The classification of local and global solutions with given holonomy groups is discussed.
\end{abstract}

PACS: 04.20.Cv, 04.60.+n, 02.40.+m

\footnotetext{
${ }^{1}$ jacobson@umdhep.umd.edu

${ }^{2}$ romano@umdhep.umd.edu
} 


\section{Introduction}

Since there is such a dearth of known observables in general relativity, any observable is worth studying. This is especially true in view of issues raised by quantum theory. For example, it is only when true observables are known that the physical inner product in Hilbert space can be constrained by reality conditions, and meaningful physical statements can be extracted from the theory. Moreover, an observable constructed entirely from the chiral spin-connection is particularly interesting because, as realized by Ashtekar, the components of this connection form a complete set of coordinates having vanishing Poisson brackets on the phase space of complexified general relativity. The corresponding quantum operators therefore commute, so an observable built purely from them may be free of operator-ordering ambiguities.

It is therefore noteworthy that the holonomy group of the chiral spin-connection is an observable in vacuum general relativity (GR). More precisely, as shown in a recent paper by Goldberg, Lewandowski, and Stornaiolo [1], the complexification of the holonomy group based at a point $*$ is preserved under time evolution. In addition, this holonomy group is invariant under spatial diffeomorphisms and $S L(2, C)$ spin-transformations that are the identity at $*$. Thus, the holonomy group qualifies as an observable once the basepoint $*$ and spin-frame at $*$ are fixed. This observable is determined by the spin-connection on an initial value hypersurface. Thus, in a hamiltonian formulation of the theory, it is determined by the phase space coordinates, without implicitly or explicitly solving the dynamics.

In this paper, the result of [1] is extended in several directions. First, the underlying reason for the time-independence of the holonomy group is traced to the self-duality of the curvature 2-form in the absence of matter. This observation reveals that the holonomy group is time-independent not only in vacuum, but also in the presence of a cosmological constant. It also shows that once matter is coupled to gravity, the "conservation of holonomy" is lost.

For a generic point in phase space, the holonomy group will be all of $S L(2, C)$. Thus, the holonomy group observable does not contain very much information about the gravitational field in general, and for this reason, it would appear not to be a very interesting observable. It is, however, better than nothing. Moreover, when the fundamental group of space is non-trivial, there is a refinement of the holonomy group observable. For each homotopy class of loops, the holonomies comprise a coset of the full holonomy group. This coset is also time-independent. 
But since the fundamental group is not, in general, invariant under large diffeomorphisms, the homotopy-class holonomy cosets are not quite observables. To obtain observables, the action of these diffeomorphisms must be factored out.

This construction mirrors that of $2+1$ dimensional gravity, where the vacuum equations imply local flatness of the $S O(2,1)$ frame-connection, and the map from homotopy classes to holonomy elements yields an observable after factoring by the mapping class group [2, 3]. Remarkably, the 3+1-dimensional homotopy observables exist even though the connection is not necessarily flat. They are non-trivial, however, only if the holonomy group is not all of $S L(2, C)$.

The rest of this paper is organized as follows: First, in section 2, we provide our alternate proof of the conservation of holonomy, showing that the result extends to the case of a cosmological constant, but not to arbitrary matter coupling. Next, in section 3 , we spell out the relation between this local result and the global statement that the holonomy group is an observable. This discussion is intended to provide an explicit treatment of some points that were implicit in [1]. In order to have the most general result, we will take care to allow for arbitrary spatial topology and arbitrary connections. In section 4 , the homotopy observable is introduced, and in section 5, we classify the cases that can potentially arise for this observable. In section 6 , the local classification of solutions with restricted holonomy algebras is given, and the global classification problem is discussed but not solved. Finally, section 7 contains a brief discussion of the results, their possible uses, and open questions. An appendix contains a proof of the reduction theorem used in section 3.

\section{Local Result: Time-independence of the connection algebra}

The local result was proved in [1] using Ashtekar's hamiltonian formulation of $\mathrm{GR}$, in a case by case analysis of the subalgebras of the Lie algebra $s l(2, C)$. It turns out to be much easier to see what is happening by taking a more 4-dimensional point of view. In what follows, we will assume that the spacetime manifold $M$ has the form $M=\Sigma \times R$ for some connected 3-manifold $\Sigma$, and that the spacetime metric is non-degenerate. Degenerate metrics were considered in [1] using the Ashtekar variables approach. In general, time independence of the holonomy group is lost when the metric is degenerate, although there are special cases for which it still holds. 
Consider a 4-dimensional left-handed spin-connection $\omega_{\mu}{ }^{A B}$ and its curvature 2 -form $R_{\mu \nu}{ }^{A B}$ at any spacetime point $(x, t)$ and in any gauge. If $\omega_{\mu}{ }^{A B}$ is the spinconnection corresponding to a vacuum solution of Einstein's equation, then the vanishing of the tracefree part of the Ricci tensor implies that the curvature 2-form $R_{\mu \nu}{ }^{A B}$ is self-dual. In coordinates that are orthonormal at a point, this means that

$$
R_{0 i}{ }^{A B}=i \epsilon^{i j k} R_{j k}{ }^{A B}
$$

where $i, j, k$ are spatial indices and 0 is timelike, and we have assumed Lorentz signature.

In a gauge with $\omega_{0}{ }^{A B}=0, R_{0 i}{ }^{A B}$ is simply the time derivative of $\omega_{i}{ }^{A B}$. If in a neighborhood of $x \in \Sigma, \omega_{i}{ }^{A B}$ initially takes values in some subalgebra of $\operatorname{sl}(2, C)$, then $R_{i j}{ }^{A B}(x)$ takes values in the same subalgebra. Thus, under time evolution according to the vacuum equations, the self-duality equation (1) shows that for some time interval, $\omega_{i}{ }^{A B}(x)$ will remain in this subalgebra in a gauge with $\omega_{0}=0$. Note that because of the factor of $i$ in equation (1), it is the complex subalgebra that is preserved. Note also that this argument actually shows that the holonomy algebra is preserved along any foliation of spacetime, not just a spacelike one.

Since the curvature 2-form $R_{\mu \nu}{ }^{A B}$ is also self-dual in the presence of a cosmological constant, the corresponding subalgebra is time-independent in a gauge with $\omega_{0}=0$ in that case as well. However, in the presence of matter, $R_{\mu \nu}{ }^{A B}$ is no longer self-dual. Then we have

$$
R_{\mu \nu}^{A B}=X^{A B C D} \Sigma_{\mu \nu C D}+\Phi^{A B C^{\prime} D^{\prime}} \bar{\Sigma}_{\mu \nu C^{\prime} D^{\prime}},
$$

where $\Sigma_{C D}=\theta_{C} C^{\prime} \wedge \theta_{D C^{\prime}}$, with $\theta_{\mu}{ }^{C C^{\prime}}$ being tetrad 1-forms. The 2-forms $\Sigma_{C D}$ are self-dual, and the conjugates $\bar{\Sigma}_{C^{\prime} D^{\prime}}$ are anti self-dual. These are independent, and together they span the 6-dimensional space of 2-forms. $\Phi^{A B C^{\prime} D^{\prime}}$ is the spinor equivalent of the tracefree part of the Ricci tensor, which according to Einstein's equation is proportional to the tracefree part of the energy-momentum tensor. Now if $R_{i j}{ }^{A B}$ takes values in some subalgebra on an initial value surface, this will in general no longer imply anything about $R_{0 i}{ }^{A B}$, since $R_{\mu \nu}{ }^{A B}$ has independent self-dual and anti self-dual parts.

\footnotetext{
${ }^{3}$ After a finite time interval has passed, it may happen that there is no longer a neighborhood of $x$ in which $\omega_{i}{ }^{A B}$ falls within the subalgebra, since curvature can propagate to $x$ from other regions. If this happens, the connection will go outside the original subalgebra 沺. Nevertheless, as will be explained in section 3 , the complexification of the full holonomy group will be conserved.
} 


\section{Global Result: The holonomy group observable}

The local result just proved states that the complex subalgebra spanned by the values of the connection 1-form $\omega_{i}{ }^{A B}(x)$ at a point $x \in \Sigma$ in a gauge with $\omega_{0}=0$ is temporarily time-independent in vacuum GR. I But this result does not directly yield an observable. First of all, "temporary" time-independence is not good enough! Moreover, the above subalgebra is not invariant under spatial diffeomorphisms, since it depends essentially arbitrarily on the choice of $x$. Both of these problems can be remedied by considering the smallest subalgebra containing the union of the subalgebras spanned by $\omega_{i}{ }^{A B}(x)$ for all $x \in \Sigma$, in some globally defined gauge.尸

The resulting union of subalgebras is still not an observable, however, because it is not invariant under arbitrary local $S L(2, C)$ transformations. Only if the resulting subalgebra happens to be an ideal will it be invariant. Thus, one way to obtain an observable is to take the smallest ideal generated by the span of $\omega_{i}{ }^{A B}(x)$ for all $x \in \Sigma$. This raises the question, what ideals of $\operatorname{sl}(2, C)$ are there? (We are interested in complex ideals, since the result of time-independence applies only to the complex subalgebra.) Unfortunately, the only ideals of $\operatorname{sl}(2, C)$ are the identity and the whole algebra, so this observable only distinguishes flat from non-flat connections.

\section{The Holonomy group}

A more interesting observable, as noted by Goldberg et al [1], is given by the holonomy group of the connection, which is defined as follows: A basepoint $* \in \Sigma$ is fixed, and one considers all closed loops in $\Sigma$ that begin and end at $*$. The holonomy $h_{\gamma}[\omega]$ of the connection 1-form $\omega$ around a loop $\gamma$ based at $*$, is the $S L(2, C)$ element determined by the parallel transport of a spin-frame (or gauge) at $*$ around the loop. If the connection is given in a global gauge, then the holonomy has the standard form, $h_{\gamma}[\omega]=\mathcal{P} \exp \oint_{\gamma} \omega$, where $\mathcal{P}$ indicates path ordering. The set $\left\{h_{\gamma}[\omega] \mid \gamma\right.$ is a loop based at $\left.*\right\}$ is a subgroup of $S L(2, C)$, called the holonomy group based at $*$. Its Lie algebra is called the holonomy algebra based at $*$.

\footnotetext{
${ }^{4}$ See the previous footnote.

${ }^{5}$ A global gauge always exists, since $S L(2, C)$ bundles over 3-manifolds are always trivial. To see why, note that the obstructions to trivializing a $G$-bundle are the homology groups $H_{k}\left(\Sigma, \pi_{k-1}(G)\right)$. The first non-trivial homotopy group of $S L(2, C)$ is $\pi_{3}$, but $H_{k}$ of a 3 -manifold $\Sigma$ vanishes for $k>3$.
} 
If the loop $\gamma$ is covered by a finite number of local gauges $\sigma_{0}, \sigma_{1}, \cdots, \sigma_{n}$, then the holonomy element can be expressed as

$$
h_{\gamma}[\omega]=\left(\mathcal{P} \exp \int_{y_{n 0}}^{*} \omega^{(0)}\right)\left(\psi_{n 0}\right)^{-1} \cdots\left(\mathcal{P} \exp \int_{y_{01}}^{y_{12}} \omega^{(1)}\right)\left(\psi_{01}\right)^{-1}\left(\mathcal{P} \exp \int_{*}^{y_{01}} \omega^{(0)}\right),
$$

where $y_{i j}$ is any point in the overlap region of the local gauges $\sigma_{i}$ and $\sigma_{j}, \omega^{(i)}$ is the connection in the gauge $\sigma_{i}$, and $\psi_{i j}$ is the gauge transformation (i.e., transition function) at $y_{i j}$ from $\sigma_{i}$ to $\sigma_{j}$. To see that (3) is the appropriate expression for the holonomy, note that under a gauge transformation at the endpoints, the parallel propagator transforms as $\mathcal{P} \exp \int_{a}^{b} \omega^{\prime}=g_{b}^{-1}\left(\mathcal{P} \exp \int_{a}^{b} \omega\right) g_{a}$. Thus, if $\sigma_{0}$ is a global gauge and if all the propagators in (3) are expressed in the gauge $\sigma_{0}$ using this transformation formula, then (3) collapses to the standard form, $\mathcal{P} \exp \oint_{\gamma} \omega^{(0)}$.

The holonomy group based at $*$ is invariant under gauge transformations that are the identity at $*$. It is also invariant under spatial diffeomorphisms that leave * fixed. This is because such diffeomorphisms merely mix up the various loops based at $*$. The holonomy group is conjugated by some group element if the gauge at * is changed or if a different basepoint and gauge are chosen [5]. This residual gauge and diffeomorphism dependence of the holonomy group is typically regarded as rather benign, since it involves only a single overall conjugation. To eliminate this dependence, it would be necessary to tie both $*$ and the gauge at $*$ to some other physical quantity.

\section{Time-independence}

Our next step is to show that the local result discussed previously implies that the complexification of the holonomy group based at $*$ is preserved under time evolution. (Here the complexification of a subgroup $H$ of $S L(2, C)$ is defined as the smallest subgroup containing $H$ and the exponential of the complexification of the Lie algebra of $H$.) The most elegant way to show this is to invoke the reduction theorem for a connection on a principal fiber bundle [5]. The reduction theorem states that the set of points that can be joined to a point $p$ in the principal fiber bundle by a horizontal curve forms a subbundle, with structure group equal to the holonomy group at $p$. (This subbundle is called the holonomy bundle through

\footnotetext{
${ }^{6}$ In the hamiltonian picture, the diffeomorphisms act on the spin-connection, which is a phase space variable, and leave the points of the submanifold $\Sigma$ fixed. But the holonomy of the transformed connection around a loop $\gamma$ equals the holonomy of the original connection around the loop that is mapped into $\gamma$ via the diffeomorphism.
} 
p.) The reduction theorem implies that $\Sigma$ can be covered by a collection of local gauge patches such that $(a)$ in each gauge patch the connection takes values in the holonomy algebra based at $*$, and $(b)$ the gauge tranformations relating the patches take values in the holonomy group based at $*$. For completeness, we give a simple proof of the reduction theorem in the appendix.

Now condition $(a)$ and the local result together imply that there exists a set of local gauge patches covering the spacetime $M=\Sigma \times R$, in which the connection remains within the original holonomy algebra based at $*$. These gauges are obtained by imposing the additional gauge condition $\omega_{0}=0$ to extend the local gauges on $\Sigma$ to the spacetime $M$. With this gauge condition, the transition functions are time independent, so condition (b) ensures that for this set of extended gauges, the transition functions all lie within the original holonomy group based at $*$. If we use these results in conjunction with expression (3) for the holonomy of the connection around a loop $\gamma$ based at $*$, we see that under time evolution, the holonomy group never goes outside the complexification of the original holonomy group. In fact, nor can the holonomy group shrink to a proper subgroup of the original group, since the Einstein equation is time reversal invariant, and the time-reversed process would violate the previous result. In other words, the complexification of the holonomy group is preserved under time evolution.

\section{Homotopy Observables}

If $\Sigma$ is not simply connected, each homotopy class of loops determines a collection of holonomy elements, and this collection forms a coset of the full holonomy group modulo its connected component. This gives rise to a refinement of the holonomy group observable, as will now be explained.

Let $\Phi$ be the full holonomy group (at a basepoint $*$ in a fixed gauge at $*$ ), and let $\Phi_{0}$ be the restricted holonomy group, i.e., the group of holonomies around contractible loops. $\Phi_{0}$ is a connected, normal subgroup of $\Phi$, and the quotient group $\Phi / \Phi_{0}$ is countable. It follows that $\Phi_{0}$ is, in fact, the same as the connected component of the identity in $\Phi$. The holonomy map from the loop group to $\Phi$ passes to a homomorphism

$$
f: \pi_{1}(\Sigma) \rightarrow \Phi / \Phi_{0}
$$

from the fundamental group $\pi_{1}(\Sigma)$ (based at $*$ ) onto the quotient of the holonomy groups. These facts are proved in The Book, ref. [5]. We shall call the homo- 
morphism $f$ the homotopy map, although a more accurate name would perhaps be "homotopic holonomy map".

It follows from the results of the previous section that the association $f$ of a homotopy class of loops with an element of $\Phi / \Phi_{0}$ is time-independent when the spin connection evolves according to the vacuum Einstein equation. $f$ is also invariant under gauge transformations that are the identity at $*$, and under diffeomorphisms that fix $*$ and are isotopic to the identity. "Large" diffeomorphisms, i.e., ones that are not isotopic to the identity, can act on $\pi_{1}(\Sigma)$ by a non-trivial automorphism, so $f$ is not, in general, invariant under these.

According to the principle that one cannot physically distinguish spacetimes that are related by a diffeomorphism, a true gravitational observable must be invariant under large diffeomorphisms.] Thus, the homotopy map is not quite an observable as it stands, even once $*$ and the gauge at $*$ are fixed. What is required is to factor out by the action of the large diffeomorphisms. This leads one to the problem of classifying such actions, which is not always an easy problem. Even if one knows all the automorphisms of $\pi_{1}(\Sigma)$, they are generally not all induced by some diffeomorphism. Thus, there is no general solution to this problem.

To illustrate what is going on, let us consider a particular case where the solution is known. For the manifold $\Sigma=S^{1} \times R^{2}$, we have $\pi_{1}(\Sigma)=Z$, the additive group of integers. This group admits only two automorphisms: the identity, and the mapping that sends $n$ to $-n$. The inversion is induced by an inversion diffeomorphism that sends $\theta$ to $-\theta$, where $\theta$ coordinatizes $S^{1}$. Thus, in this case, we cannot associate an observable with an individual homotopy class, but rather with a pair of such classes. The observable in question is the corresponding pair of elements of $\Phi / \Phi_{0}$.

\section{Classification}

In this section the classification of possible homotopy maps $f: \pi_{1}(\Sigma) \rightarrow \Phi / \Phi_{0}$ will be obtained. This can be accomplished in the following three stages:

1. classify $\Phi_{0}$;

\footnotetext{
${ }^{7}$ This point of view has received support from an exact calculation of point-particle scattering in $2+1$-dimensional quantum gravity [6]. In this setting, the large diffeomorphisms comprise a braid group, and Carlip showed in [6] that the correct semi-classical limit is obtained only if one demands that the quantum state be invariant under this braid group.
} 
2. classify $\Phi$ in which $\Phi_{0}$ is a normal subgroup, with $\Phi / \Phi_{0}$ countable;

3. determine which of these $\Phi$ 's can arise as holonomy groups for a given 3manifold $\Sigma$.

In the next section, we shall add the requirement that the connection occurs in a solution to the Einstein equation.

\section{Classification of $\Phi_{0}$}

The only condition on the restricted holonomy group $\Phi_{0}$ is that it be a connected Lie subgroup of $S L(2, C)$. In fact, we are interested in complex subgroups, since it is these that are preserved in time. These are determined by complex Lie subalgebras of $\operatorname{sl}(2, C)$. As noted in [1], all such subalgebras are equivalent via conjugation to one of the following:

$$
s l(2, C), \quad \mathcal{A}(+, 3), \quad \mathcal{A}(+), \quad \mathcal{A}(3), \quad 0 .
$$

Here $\mathcal{A}(+, 3), \mathcal{A}(+)$, and $\mathcal{A}(3)$ denote the subalgebras generated by $\left\{\tau_{+}, \tau_{3}\right\}, \tau_{+}$, and $\tau_{3}$, where

$$
\tau_{+}=\left(\begin{array}{ll}
0 & 1 \\
0 & 0
\end{array}\right) \quad \text { and } \quad \tau_{3}=\left(\begin{array}{cc}
1 & 0 \\
0 & -1
\end{array}\right) .
$$

The corresponding groups $\Phi_{0}$ are $S L(2, C)$, the upper triangular subgroup $G(+, 3)$, the upper triangular subgroup with unit diagonal $G(+)$, the diagonal subgroup $G(3)$, and the identity subgroup. The last case corresponds to locally flat connnections.

\section{Classification of $\Phi$}

As stated in section $4, \Phi_{0}$ must be normal in $\Phi$. This is because, in the loop group, the conjugate of a trivial loop by any other loop is again trivial. Thus, for each case above, we shall find the largest subgroup of $S L(2, C)$ in which $\Phi_{0}$ is normal (i.e., the normalizer of $\Phi_{0}$ ), and then examine its subgroups. Only those subgroups $\Phi$ for which the quotient $\Phi / \Phi_{0}$ is countable can arise as possible holonomy groups, since the homotopy map $f$ is onto, and the fundamental group $\pi_{1}(\Sigma)$ is countable.

$\Phi_{0}=S L(2, C):$ We have $\Phi=\Phi_{0}$ and $\Phi / \Phi_{0}=\{i d\}$

$\Phi_{0}=G(+, 3)$ : One easily sees that $G(+, 3)$ is its own normalizer. Thus, again $\Phi=\Phi_{0}$ and $\Phi / \Phi_{0}=\{i d\}$. 
$\Phi_{0}=G(+)$ : The normalizer of $G(+)$ is $G(+, 3)$. The subgroups of $G(+, 3)$ that contain $G(+)$ are the groups of upper triangular matrices with unit determinant whose diagonal components form a subgroup $K$ of the non-zero complex numbers $C^{*}$. We will call these groups $G(+, K)$. For $\Phi=G(+, K)$, one finds $\Phi / \Phi_{0}=K$. Thus, the possible $\Phi$ 's in this case are in one to one correspondence with countable subgroups of $C^{*}$. All such subgroups are products of finite or infinite cyclic groups.

$\Phi_{0}=G(3)$ : The normalizer of $G(3)$ is the group consisting of all diagonal and purely off-diagonal matrices (with unit determinant). We shall call this group $G\left(3, Z_{2}\right)$. The only proper subgroup of $G\left(3, Z_{2}\right)$ that contains $G(3)$ is $G(3)$ itself, so $\Phi$ must be either all of $G\left(3, Z_{2}\right)$ or $G(3)$. In the former case we have $\Phi / \Phi_{0}=Z_{2}$, where $Z_{2}$ is the group of order 2 . In the latter case, we have $\Phi / \Phi_{0}=\{i d\}$.

$\Phi_{0}=\{i d\}$ : The normalizer is all of $S L(2, C)$. Thus, $\Phi$ can be any countable subgroup of $S L(2, C)$, and $\Phi / \Phi_{0}=\Phi$.

$\Phi$ as a holonomy group

The previous subsection provides a group-theoretic classification of those $\Phi$ that may arise as the holonomy group of an $S L(2, C)$ connection on a 3 -manifold $\Sigma$. If $\Sigma$ is fixed, not all of these cases will generally be realized by a connection. A necessary condition that $\Phi$ arise as a holonomy group is that there exist a homomorphism (4) from $\pi_{1}(\Sigma)$ onto $\Phi / \Phi_{0}$. In fact, this is also a sufficient condition, as will now be argued.

Let $f: \pi_{1}(\Sigma) \rightarrow \Phi / \Phi_{0}$ be such a homomorphism, which we assume exists. Choose a discrete subgroup $D \subset \Phi$ consisting of one element from each coset of $\Phi / \Phi_{0}$, so that $\Phi / \Phi_{0} \rightarrow D$ is an isomorphism of groups. Then we can define a locally flat connection with holonomy group $D$ by lifting $\pi_{1}(\Sigma) \stackrel{f}{\rightarrow} \Phi / \Phi_{0} \rightarrow D$ to a holonomy map from the loop group to $D$. This is because any homomorphism from the loop group to the structure group, that vanishes on so-called "thin" loops, determines a principal bundle and connection up to gauge transformations [7] .9] To obtain a connection with holonomy group $\Phi$, one need only make a generic deformation of the connection 1 -form taking values in the Lie algebra of $\Phi_{0}$. The curvature will

\footnotetext{
${ }^{8}$ This is possible for all $\Phi$ associated with $S L(2, C)$. In general, it is possible if and only if $\Phi$ is isomorphic to the semidirect product of $\Phi / \Phi_{0}$ with $\Phi_{0}$.

${ }^{9} \mathrm{~A}$ smoothness condition must also be assumed in order that the bundle and connection be differentiable. This condition is satisfied for the holonomy map defined above.
} 
then "fatten" the holonomy of a homotopy class associated with $g \in D$ to the corresponding coset $g \Phi_{0}$. To be fancy, one can invoke the Ambrose-Singer theorem [5], which states that the holonomy algebra at a point $p$ in the principal fiber bundle is generated by the spans of the curvature 2-forms on the holonomy bundle through $p$. Thus, any deformation of the connection for which the local curvature spans the Lie algebra of $\Phi_{0}$ will yield the holonomy group $\Phi$.

Do manifolds $\Sigma$ and (onto) homomorphisms $f: \pi_{1}(\Sigma) \rightarrow \Phi / \Phi_{0}$ always exist? The answer is yes. For suppose that the fundamental group of $\Sigma$ is a free group on $n$ generators, with $n$ greater than or equal to the number of generators of $\Phi / \Phi_{0}$. (For instance, $\Sigma$ could be a 3 -sphere with $n$ handles.) Then since there are no relations among the generators of $\pi_{1}(\Sigma)$, one can define $f$ by freely assigning at least one generator of $\pi_{1}(\Sigma)$ to each generator of $\Phi / \Phi_{0}$, and then extending this map to a homomorphism.

Thus, each $\Phi$ classified in the previous subsection can arise as the holonomy group of an $S L(2, C)$ connection on some 3-manifold $\Sigma$. Indeed, all the $\Phi$ 's can occur on the same manifold $\Sigma$, provided the fundamental group of $\Sigma$ is free and has an infinite number of generators (although this probably entails $\Sigma$ being noncompact).

\section{Examples}

In this subsection, some examples of $S L(2, C)$ connections that have disconnected holonomy groups will be given. These serve to make the preceeding classification more concrete. As noted above, the only disconnected holonomy groups for $S L(2, C)$ connections that are not locally flat are $(i) \Phi=G(+, K)$ where $K$ is any countable subgroup of $C^{*}$, and $(i i) \Phi=G\left(3, Z_{2}\right)$. We now take these in turn.

(i) $\Phi=G(+, K)$

If in a global gauge the connection $\omega$ is of the form $\omega=\omega^{+} \tau_{+}+\omega^{3} \tau_{3}$, then the holonomy $h_{\gamma}[\omega]$ around a loop $\gamma$ is an element of $G(+, 3)$ given by

$$
h_{\gamma}[\omega]=\left(\begin{array}{cc}
\exp \oint_{\gamma} \omega^{3} & z_{\gamma} \\
0 & \exp \left(-\oint_{\gamma} \omega^{3}\right)
\end{array}\right) .
$$

Here

$$
z_{\gamma}=\left(\exp \oint_{\gamma} \omega^{3}\right) \oint_{\gamma} e^{-2 \int_{0}^{s} \omega^{3}} \omega^{+}
$$

and $s$ denotes a parameter along the loop $\gamma$. In order to restrict the connection so that $\Phi_{0} \subset G(+)$, it is sufficient that the 1 -form $\omega^{3}$ be closed, so that $\oint_{\gamma} \omega^{3}=0$ 
when $\gamma$ is homotopic to the identity. For a generic $\omega^{+}, \Phi_{0}$ will then comprise all of $G(+)$.

Now let $K$ be a countable subgroup of $C^{*}$, and let $\left\{a_{i}\right\}$ denote a (possibly infinite) set of non-zero complex numbers that generate $K$. Suppose that the number of generating elements of $K$ is less than or equal to the dimension of the first homology group of $\Sigma, H_{1}(\Sigma)$, and let $\left\{\gamma_{i}\right\}$ denote a generating set of 1-cycles for $H_{1}(\Sigma) .0$ To arrange for the holonomy group to be $G(+, K)$, it suffices to impose the further restrictions that the closed 1-form $\omega^{3}$ satisfy $\oint_{\gamma_{i}} \omega^{3}=\ln a_{i}$. If there are more generators $\gamma_{i}$ than $a_{i}$, then we can require that $\oint_{\gamma_{i}} \omega^{3}=0$ for the additional $\gamma_{i}$. The existence of such a closed 1 -form is guaranteed by the de Rahm theorem (see, e.g., [8]), which establishes the duality of the first homology and cohomology groups. Note that by defining $\omega$ in this fashion, we have effectively encoded all the "extra holonomy" in the $\tau_{3}$ part of the connection.

For example, suppose that the manifold $\Sigma=T^{2} \times R$, and $K$ is generated by two generic non-zero complex numbers $a_{1}$ and $a_{2}$. $K$ is then isomorphic to $Z \times Z$, unless $a_{1}$ or $a_{2}$ is a root of unity. The manifold $T^{2} \times R$ has both its fundamental and first homology groups equal to $Z \times Z$. Let us take $\gamma_{1}=\left(\theta_{1}, 0,0\right)$ and $\gamma_{2}=\left(0, \theta_{2}, 0\right)$ as the two generating loops on $\Sigma$, where $\theta_{1}, \theta_{2} \in[0,2 \pi)$ coordinatize the torus. Then the above discussion shows that the connection

$$
\omega=\omega^{+} \tau_{+}+\frac{1}{2 \pi}\left(\ln a_{1} d \theta_{1}+\ln a_{2} d \theta_{2}\right) \tau_{3}
$$

has holonomy $\Phi=G(+, Z \times Z)$, for $\omega^{+}$any non-vanishing 1-form on $R$. In particular, one could choose $\omega^{+}=d z$, where $z$ is a coordinate on $R$ that is constant on $T^{2}$. In this case, the curvature that "fills out" $\Phi_{0}=G(+)$ comes from the commutator $\left[\tau_{3}, \tau_{+}\right]=2 \tau_{+}$.

The connections above have been defined in a global gauge, and take values in the algebra $\mathcal{A}(+, 3)$, although the holonomy algebra itself is $\mathcal{A}(+)$. This means that in a covering family of radial gauges (see section 4 and the Appendix), the connection takes values only in $\mathcal{A}(+)$, and the gauge transition functions take values in $G(+, 3)$. This observation may be important if one tries to construct solutions with non-trivial homotopy observables.

(ii) $\Phi=G\left(3, Z_{2}\right)$

Instead of working in a global gauge like we did in the previous example, we

\footnotetext{
${ }^{10}$ We need to introduce homology groups in order to later appeal to de Rahm's theorem.
} 
will take $\Sigma=S^{1} \times S^{2}$, and cover $\Sigma$ with two local gauges $\sigma_{0}$ and $\sigma_{1}$ defined on $U_{0} \times S^{2}$ and $U_{1} \times S^{2}$. (Here $U_{0}$ and $U_{1}$ cover $S^{1}$, with two overlap regions.) These gauges are chosen so that they agree on one overlap region, but disagree on the other, where they are related by the constant transition function $\psi_{01}=\left(\begin{array}{cc}0 & 1 \\ -1 & 0\end{array}\right)$.

Suppose that in each local gauge the connection is of the form $\omega=\omega^{3} \tau_{3}$. Then since the transition function $\psi_{01}$ lies in the normalizer of $G(3)$, we have $\Phi_{0} \subset G(3)$. For generic $\omega^{3}, \Phi_{0}$ will comprise all of $G(3)$. To see that $\Phi=G\left(3, Z_{2}\right)$, let $\gamma$ be a loop that wraps once around $S^{1}$ and traverses each overlap region once. Since $\psi_{01}$ lies in the normalizer of $G(3)$, equation (3) shows that the holonomy $h_{\gamma}[\omega]$ is a product of $\psi_{01}$ with some element of $G(3)$. This product belongs to the non-identity coset in $G\left(3, Z_{2}\right)$. Since $\Phi_{0}$ is all of $G(3), \Phi$ is thus all of $G\left(3, Z_{2}\right)$.

Since the local gauge $\sigma_{1}$ can be deformed to agree with $\sigma_{0}$ in both overlap regions, one can write the connection (defined above) in a global gauge without the need of transition functions. But in order to do this, one would have to perform a gauge transformation that is the identity in one overlap region, and takes $\psi_{01}$ to the identity in the other. This gauge transformation would necessarily involve group elements of the form $\exp \left(\alpha \psi_{01}\right)$, where $\alpha \in[0,2 \pi)$. That the holonomy group equals $G\left(3, Z_{2}\right)$ would no longer be manifest if the connection were expressed in this global gauge.

\section{The Einstein equation}

A classical observable is a function on the space of solutions to the equations of motions modulo gauge transformations. Thus, only those holonomy groups that arise in a Lorentzian solution to the Einstein equation are relevant. It is thus of interest to determine which holonomy groups classified above actually occur in solutions, and to characterize those solutions as far as possible.

In the first subsection, Local solutions, we will consider only the local restrictions

\footnotetext{
${ }^{11}$ This is a special case of a general result: If a connection takes values in a subalgebra $h$ in each gauge patch, and if the transition functions lie in the normalizer of the corresponding subgroup $H$, then the holonomy around any contractible loop lies in $H$.

${ }^{12}$ The relevance of this issue goes beyond the holonomy group considerations of the present paper. It would seem to be of fundamental interest to the Ashtekar variables program to have the general answer, in order to know which connections actually lie in the reduced phase space. In the quantum theory, those connections that are not in the reduced phase space should be associated with zero "measure" in a functional integral or Hilbert space inner product.
} 
imposed by the Einstein equation, for each of the possible holonomy subalgebras. These local considerations do not suffice, however, to establish (or classify) the existence of globally regular solutions with a given holonomy group. One would ideally like to classify such solutions for each 3-manifold $\Sigma$ and each holonomy group $\Phi$. In the second subsection, Global considerations, this problem will be discussed. We have no complete answer to this global problem as of now. However, some partial results will be given.

\section{Local solutions}

If the holonomy algebra of a spacetime is not all of $\operatorname{sl}(2, C)$, then it is contained in $\mathcal{A}(+, 3)$, and there is a covariantly constant null direction in the spacetime. The vacuum solutions with this property are the Goldberg-Kerr solutions [9]. These have a line element that can be locally written in the form [1]

$$
d s^{2}=2 d z d \bar{z}-2 d u(d r+W d z+\bar{W} d \bar{z}+H d u)
$$

where

$$
\begin{aligned}
& H=\frac{1}{2}\left(W_{\bar{z}}+\bar{W}_{z}\right) r+H^{0}, \\
& H^{0}=\operatorname{Re}\left[\left(W W_{\bar{z}}+W_{u}\right) z+h(z, u)\right],
\end{aligned}
$$

and $W(\bar{z}, u), h(z, u)$ are arbitrary functions holomorphic in $\bar{z}$ and $z$, respectively. (Subscripts denote partial differentiation.)

If the holonomy algebra is $\mathcal{A}(+)$, then there is a covariantly constant null vector, and the only vacuum solutions with this property are the $p p$ waves [10]. These correspond to the Goldberg-Kerr solutions above with $W=0$. If the holonomy algebra is $\mathcal{A}(3)$, then as shown in [1] (using Ashtekar's form of the initial value constraints), the only vacuum solution is flat space, so the algebra is actually trivial. Thus, the only vacuum solutions with non-trivial homotopy map are spacetimes that are locally $p p$ waves with holonomy group $G(+, K)$, and locally flat spacetimes.

In the presence of a cosmological constant, the locally flat solutions are excluded. In addition, Boström has shown [11] (again, using the Ashtekar constraints) that there are no solutions with holonomy algebra $\mathcal{A}(+)$. To our knowledge, the solutions with non-zero cosmological constant and holonomy algebra equal to $\mathcal{A}(+, 3)$ have not been classified.

The remaining case to consider is that of the holonomy algebra $\mathcal{A}(3)$. It turns out that this case is allowed in the presence of a cosmological constant $\Lambda$, although 
there is locally only one solution for each value of $\Lambda$. To see this, note first that there are now two covariantly constant null directions, say those of the vectors $l^{\mu}$ and $n^{\mu}$. It is easy to verify that $l^{\mu}$ and $n^{\mu}$ are both hypersurface orthogonal and surface-forming. We can therefore introduce null coordinates $u, v$ and complex spatial coordinates $z, \bar{z}$ for which the line element takes the form

$$
d s^{2}=|f|^{2}(u, v, z, \bar{z}) d z d \bar{z}-g(u, v, z, \bar{z}) d u d v .
$$

The condition that the holonomy algebra is $\mathcal{A}(3)$ implies that the connection generates only boosts in the $u, v$-plane and rotations in the complex $z$-plane, which in turn implies $|f|^{2}=|f|^{2}(z, \bar{z})$ and $g=g(u, v)$. The spacetime is thus locally a direct product of 2-dimensional Euclidean and Lorentzian spaces.

Applying the Einstein equation with cosmological constant $\left(R_{\mu \nu}=\Lambda g_{\mu \nu}\right)$, we find that the 2-dimenstional sections are spaces of the same constant curvature. If $\Lambda=0$ we have flat space; if $\Lambda>0$ we have the product of a 2 -sphere with 2-dimensional de Sitter space; and if $\Lambda<0$ we have the product of a 2-hyperboloid with 2-dimensional anti-de Sitter space. These solutions with $\Lambda \neq 0$ are the Nariai solutions [12].

Thus the only solutions with $\Lambda \neq 0$ and non-trivial homotopy map are locally the Nariai solutions, with holonomy group $G\left(3, Z_{2}\right)$.

\section{Global considerations}

There are two types of global issues to consider: classification of globally regular solutions with a given holonomy algebra, and classification of globally regular solutions with a given disconnected holonomy group.

Let us consider first the latter question, in the case where the connection is locally flat. For locally flat connections, the problem is closely analogous to one that has been answered recently for flat $S O_{0}(2,1) \cong P S L(2, R)$ connections on 2-manifolds, $\Sigma^{2}$ [13, 14]. In that case, if the genus of $\Sigma^{2}$ is greater than one, a compatible triad for which $\Sigma^{2}$ is spacelike exists if and only if the homotopy map is a discrete embedding of $\pi_{1}\left(\Sigma^{2}\right)$ into $S O(2,1)$. The $3+1$-dimensional case is somewhat different, not only because of the added dimension, but also because one is specifying just the self-dual part of the 4-dimensional spin-connection. Nevertheless, according to Carlip [15], a similar result holds. Namely, if $G$ is a discrete subgroup of $S L(2, C)$, then $G$ acts properly discontinuously on the hyperboloids of constant proper time that foliate the interior of a light cone $X$ in $3+1$ dimensional 
Minkowski spacetime. The quotient of $X$ by $G$ is a flat spacetime with induced spin connection whose holonomy group is $G$. The question of whether a flat spacetime with a discrete holonomy group must arise in this manner remains open, as does the question of whether the holonomy group must be discrete.

The Nariai solutions mentioned above for $\Lambda \neq 0$ are the only solutions with holonomy algebra $\mathcal{A}(3)$. There are various globally regular forms of these metrics, obtained by identifications. For example, for $\Lambda<0$, quotients of the hyperbolic 2 -space will yield the Teichmüller spaces of metrics on genus $g \geq 2$ surfaces. The holonomy group of all of these spacetimes will be $G(3)$.

Is it is possible to patch together local Nariai metrics in such a way as to obtain globally regular solutions with the disconnected holonomy group $G\left(3, Z_{2}\right)$ ? The answer seems to be no. For instance, consider the $\Lambda>0$ Nariai solution and the $\Phi=G\left(3, Z_{2}\right)$ example of section 5 . If $\Sigma$ of that example is identified with a spatial

slice of the Nariai solution, then the $S L(2, C)$ transition function $\psi_{01}=\left(\begin{array}{cc}0 & 1 \\ -1 & 0\end{array}\right)$ corresponds to a rotation through $90^{\circ}$ in a plane orthogonal to the $S^{2}$-tangent plane. If we were to try patching together local solutions using such a transition function, we would "mix up" the direct product structure of the Nariai metric. The transition function thus could not be induced by an isometric coordinate tranformation, so such a patching seems to be impossible.

Finally, consider the solutions that have holonomy algebra $\mathcal{A}(+, 3)$, that is, the Goldberg-Kerr solutions (7). The 2-dimensional spaces $r, u=$ const are flat, so if orientable, they must be the plane, the cylinder, or the torus $T^{2}$. Global regularity imposes severe restrictions on the $z$-dependence of the holomorphic functions $W$ and $h$ in the line element. For the $T^{2}$ case, the only holomorphic function is a constant, so $W(\bar{z}, u)=W(u)$ and $h(z, u)=h(u)$. In fact, $W$ must also be independent of $u$, since otherwise the linear term in $z$ in $H^{0}$ will not be globally extendible. The flat metric on the torus can have any Teichmüller parameters.

As discussed in the previous subsection, the $p p$ wave solutions $(W=0)$ have holonomy algebra $\mathcal{A}(+)$. Are there any gobally defined solutions that are locally $p p$ waves, and that have a disconnected holonomy group of the form $G(+, K)$ ? That is, can they support global holonomy in a discrete subgroup of $G(3)$ ? We do not know the answer to this question. However, we can offer the following remark.

Recall that the homotopy map $\pi_{1}(\Sigma) \rightarrow \Phi / \Phi_{0}=K$ is onto, so a non-trivial $K$ requires a non-trivial $\pi_{1}(\Sigma)$. For instance, if $\Sigma=T^{2} \times R$, then $K$ can have at most two generators. One might think that non-trivial Teichmüller parameters on $T^{2}$ 
would in general lead to global holonomy of the form $G(+, Z \times Z)$, but this is not the case. In 2+1-dimensions, Carlip [3] has shown explicitly how the Teichmüller parameters are expressed in terms of the generators of two commuting $\operatorname{ISO}(2,1)$ holonomies. The homogeneous parts of these holonomies are boosts in directions tangent to the torus. Embedding Carlip's analysis in 3+1-dimensions, we find that these boosts cannot be identified with boosts in $G(3)$, because the latter boost in the spatial direction orthogonal to the torus. ${ }^{13}$ Thus, to obtain $p p$ wave solutions with global holonomy in $G(3)$, it is evidently necessary to patch together local solutions with transition functions in $G(3)$. We do not know whether or not this is possible.

\section{Discussion}

The conservation of the holonomy group (or, more generally, the homotopy map) of the chiral spin-connection in vacuum GR may turn out to have interesting applications. At the classical level, it provides a global constraint on evolution of initial data, that can only be violated due to the occurence of a Cauchy horizon or singularity. Put differently, data sets with different holonomy groups cannot be cobordant in a regular vacuum solution.

For use in the quantum theory, the major drawback is probably that the holonomy group is all of $S L(2, C)$ for a generic solution, so the holonomy group observable does not distinguish typical solutions. However, in a "midi-superspace" consisting of connections with holonomy less than all of $S L(2, C)$, it would be relatively more significant. It might be interesting to formulate a midi-superspace quantization of general relativity along these lines, as an alternative to the usual truncations of the theory.

That the holonomy group is not conserved in the presence of matter couplings is, of course, another limitation in its applicability. One way to evade this limitation would be to model matter by vacuum configurations such as the Einstein-Rosen bridge of the extended Schwarzschild solution. However, since the Schwarzschild solution has holonomy group equal to all of $S L(2, C)$, this is not so useful. Another

\footnotetext{
${ }^{13}$ The null rotations in $G(+)$ are generated by $\tau_{+}=\left(\tau_{1}+i \tau_{2}\right) / 2$. In an adapted spin-frame, these spin-transformations stabilize the covariantly constant null vector, which is orthogonal to the torus. Therefore, in the adapted spin-frame, it is the 1- and 2-axes that are tangent to the torus. The 3 -axis is thus orthogonal to the torus.
} 
option would be to couple gravity to one-dimensional string-like matter. Then the energy-momentum tensor would vanish everywhere except on the world sheets, where it would be singular. If the worldsheets are removed from the spacetime, what remains is a vacuum solution, so the holonomy group should be conserved. For special string configurations, the holonomy group can be less than all of $S L(2, C)$. If the strings are knotted or linked, interesting homotopy groups can arise, allowing for a rich array of holonomy groups and their associated homotopy maps.

\section{Appendix: Reduction Theorem}

The reduction theorem played such a crucial role in establishing the timeindependence of the holonomy group that it seems worthwhile to illustrate the logic underlying this theorem. Since the principal bundle point of view is not familiar to all physicists, we shall just give a "low brow" proof that $\Sigma$ can be covered by a collection of local gauge patches such that $(a)$ in each gauge patch the connection takes values in the holonomy algebra based at $*$, and $(b)$ the gauge tranformations relating the patches take values in the holonomy group based at $*$. This result is valid for any $G$-bundle over any connected $n$-manifold $\Sigma$.

The argument is simplest when $\Sigma$ is contractible, because then we can construct a single, globally defined radial gauge based at $*$, in which the connection takes values in the holonomy algebra based at $*$. This is done as follows: Choose an arbitrary family of curves through $*$ that never cross and that fill all of space. Then choose an arbitrary gauge at $*$, and carry it out to the rest of $\Sigma$ by parallel transport along these curves. This defines a global gauge, in which the component of the connection along these curves vanishes. This means that if a loop is formed by segments of two of these curves joined by a transverse piece, the only non-trivial contribution to the holonomy will come from the transverse piece. If the transverse piece is infinitesimal, this contribution to the holonomy differs from the identity by a term proportional to the connection. Thus, in a radial gauge based at $*$, the connection takes values in the holonomy algebra based at $*$.

If $\Sigma$ is not contractible, then a global radial gauge does not exist. However, we can cover $\Sigma$ by a collection of local radial gauge patches satisfying conditions $(a)$ and $(b)$ as follows: Choose a fiducial gauge at $*$, and carry it out to a collection of points $\left\{x_{i}\right\}$ (whose local gauge patches will cover $\Sigma$ ) by parallel transport along a collection of curves $\left\{\gamma_{i}\right\}$. At each $x_{i}$, extend the resulting gauge to a local radial 
gauge $\sigma_{i}$, with one of the radial curves being $\gamma_{i}^{-1}$ (the curve $\gamma_{i}$ traversed in the opposite direction). All of the local gauges $\sigma_{i}$ cover the point $*$ and agree with the fiducial gauge there. Since the component of the connection along all the $\gamma_{i}$ vanishes in the gauge $\sigma_{i}$, the holonomy group based at $x_{i}$ in the gauge $\sigma_{i}$ coincides with the holonomy group based at $*$ in the fiducial gauge. In fact, the holonomy group based at any point $y$ in any local gauge $\sigma_{i}$ agrees with the holonomy group based at $*$ in the fiducial gauge. This is because $y$ and $x_{i}$ are always joined by some radial curve $\gamma_{i y}$ of the gauge $\sigma_{i}$, so the component of the connection along the combined curve $\gamma_{i} \gamma_{i y}$ (which connects $*$ to $y$ ) vanishes.

To show that condition $(a)$ holds, we adopt the collection of local radial gauges $\left\{\sigma_{i}\right\}$ defined above, and apply the argument used previously in the contractible case, but now with the holonomy based at $x_{i}$. In this set of gauges, the connection indeed takes values in the holonomy algebra based at $*$. To show that condition $(b)$ holds, we consider any point $y$ in the overlap of two gauges $\sigma_{i}$ and $\sigma_{j}$. The gauge transformation at $y$ from the gauge $\sigma_{i}$ to the gauge $\sigma_{j}$ is given by the holonomy at

$y$ around the loop $\gamma_{y}:=\gamma_{y i} \gamma_{i}{ }^{-1} \gamma_{j} \gamma_{j y}$, evaluated in the gauge $\sigma_{i}$. But as explained above, the holonomy group based at $y$ in the gauge $\sigma_{i}$ coincides with the holonomy group based at $*$ in the fiducial gauge. Thus, the gauge transformation is indeed an element of the holonomy group based at $*$.

\section{ACKOWLEDGEMENTS}

We are grateful to Robert Bryant, Steve Carlip, Bill Goldman, Jerzy Lewandowski, Charlie Misner, and Chris Stark for helpful conversations. This research was supported in part by NSF grant PHY91-12240. 


\section{References}

[1] J.N. Goldberg, J. Lewandowski, and C. Stornaiolo, "Degeneracy in Loop Variables," Syracuse University preprint (1991), to appear in Commun. Math. Phys.

[2] E. Witten, Nucl. Phys. B311, 46 (1988).

[3] S. Carlip, Phys. Rev. D42, 2647 (1990).

[4] J. Lewandowski, personal communication.

[5] S. Kobayashi and K. Nomizu, Foundations of Differential Geometry, Volume I (Interscience, New York, 1963).

[6] S. Carlip, Nucl. Phys. B324, 106 (1989).

[7] See, for example, J.W. Barrett, Int. J. Theor. Phys. 30, 1171 (1991), and references therein.

[8] Y. Choquet-Bruhat, C. DeWitt-Morette, and M. Dillard-Bleick, Analysis, Manifolds and Physics (North-Holland, Amsterdam, 1987), p. 226 .

[9] J.N. Goldberg and R.P. Kerr, J. Math. Phys. 2, 327 (1961); Ibid, 2, 332 (1961).

[10] D. Kramer, H. Stephanie, E. Herlt, and M. MacCallum, Exact Solutions of Einstein's Field Equations (Cambridge, London, 1980), p. 233.

[11] O. Boström, "Degeneracy in Loop Variables; Some Further Results," Institute of Theoretical Physics Göteborg preprint ITP 92-42 (1992).

[12] H. Nariai, Sci Rep. Tohuko Univ. Series 1 34, 160 (1950). Ibid, 35, 62 (1951).

[13] G. Mess, "Flat Lorentz Spacetimes," Mathematical Sciences Research Institute preprint (1989); "Lorentz Spacetimes of Constant Curvature," Institut des Hautes Etude Scientifiques preprint IHES/M/90/28 (1990). 
[14] S. Carlip, Class. Quantum Grav. 8, 5, (1991).

[15] S. Carlip, personal communication. 